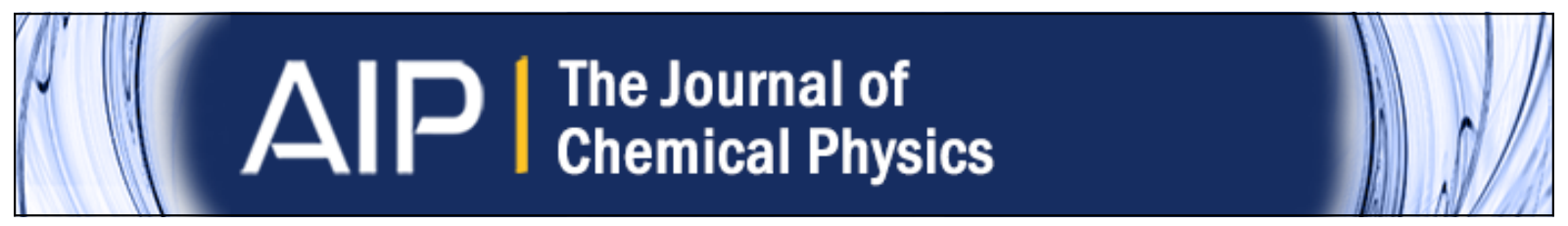

Single center method: A computational tool for ionization and electronic excitation studies of molecules

Ph. V. Demekhin, A. Ehresmann, and V. L. Sukhorukov

Citation: The Journal of Chemical Physics 134, 024113 (2011); doi: 10.1063/1.3526026

View online: http://dx.doi.org/10.1063/1.3526026

View Table of Contents: http://scitation.aip.org/content/aip/journal/jcp/134/2?ver=pdfcov

Published by the AIP Publishing

Advertisement:

AlP Re-register for Table of Content Alerts

Create a profile.

Sign up today! 


\title{
Single center method: A computational tool for ionization and electronic excitation studies of molecules
}

\author{
Ph. V. Demekhin, ${ }^{1, \text { a) }}$ A. Ehresmann, ${ }^{2}$ and V. L. Sukhorukov ${ }^{1}$ \\ ${ }^{1}$ Rostov State University of Transport Communications, 344038 Rostov-on-Don, Russia \\ ${ }^{2}$ Institute of Physics and CINSaT, University of Kassel, D-34132 Kassel, Germany
}

(Received 5 October 2010; accepted 22 November 2010; published online 13 January 2011)

\begin{abstract}
We discuss the recent progress in the development of the single center (SC) method for computation of highly-delocalized discrete and partial photoelectron wave continuous functions of molecules. Basic equations of the SC method are presented, and an efficient scheme for the numerical solution of a system of coupled Hartree-Fock equations for a photoelectron is described. Several illustrative applications of the method to photoionization and electronic excitation processes in diatomic molecules are considered. Thereby, we demonstrate its potential for theoretically studying angularly resolved molecular photoionization processes. () 2011 American Institute of Physics. [doi:10.1063/1.3526026]
\end{abstract}

\section{INTRODUCTION}

The theoretical investigation of photoionization and electronic excitation processes of molecules is a complex problem, requiring precise molecular orbitals (MOs) of the molecular ion core and the electron in discrete or continuous spectra. One of the most accurate many-center representations of a molecular orbital as the linear combination of atomic orbitals (MO LCAOs), primarily intended for the study of the lowest molecular excited states, allows one to calculate MOs of the core, valence, and first unoccupied shells with very high accuracy. The MO LCAO representation, however, is restricted to studying highly excited delocalized states in discrete and continuous spectra of molecules. Therefore, considerable efforts have been invested in the last decades to the development of theoretical methods and computational approaches to solve the electron continuum problem in molecules.

Several alternative approaches have been applied for computing molecular Auger decay rates, requiring accurate continuous wave functions for the outgoing electron. Among them are numerous scattering methods ${ }^{1-6}$ or the Stieltjes imaging technique. ${ }^{7-12}$ These approaches are, however, restricted to the calculation of the total decay rates with respect to the final Auger continuous chanels and are not designed for the computation of partial photoelectron waves. The latter difficulties are absent in the multichannel Schwinger scattering methods, ${ }^{13-17}$ where the Auger continuous channels are the solutions of the Lippmann-Schwinger equation in a numerical basis set optimized in a model Hamiltonian ${ }^{13-15}$ or in a basis set of atom-centered Gaussian functions. ${ }^{16,17}$ Subsequent asymptotic one-center extrapolation of the continuous channels via partial electron waves with given angular momentum

a) Present address: Theoretische Chemie, Physikalisch-Chemisches Institut, Universität Heidelberg, Im Neuenheimer Feld 229, D-69120 Heidelberg, Germany. Electronic mail: phd@rgups.ru and philipp. demekhin@pci.uni-heidelberg.de. $\ell$ and projection $m$ also allows calculations of electron angular distributions.

Another comparatively simple method for the computation of partial decay amplitudes and electron partial waves, proposed by Siegbahn et al. ${ }^{18}$ and applied widely nowadays, ${ }^{19-23}$ is the one-center approximation. It utilizes photoelectron continuous partial waves computed in the field of one atomic center at which the Auger decay happens, neglecting, therefore, interatomic and molecular field effects in the continuum. It describes rather satisfactorily solid-angleaveraged intensities of the Auger spectrum ${ }^{22}$ and even angularly resolved intensities. ${ }^{23}$ The latter approximations are absent in the generalization of the atomic random-phaseapproximation (RPA) for diatomic molecules. ${ }^{24-26}$ According to this method, the Hartree-Fock (HF) zero order basis set of one-particle wave functions containing both discrete excited states and the continuous spectrum is calculated numerically in prolate spheroidal coordinates in the field of a frozen core of a singly charged ion. This basis set is then used for the RPA calculations, accounting for intershell correlations. Since basis continuous wave functions are sought as the solutions with given angular momentum $\ell$ and its projection $m$ (partial electron waves), this method is most suited for the study of angularly resolved decay spectra of molecules. ${ }^{25,26}$

The present paper is focused on the recent developments of the alternative approach for studying molecular photoabsorption, which is known as the single center (SC) method, ${ }^{27,28}$ and which was among the first computational approaches for molecular wave function calculations. ${ }^{27} \mathrm{Be}-$ cause of the breakthrough in the development of many-center MO LCAO computational approaches in the middle of the last century, the use of the SC method was reduced. Its comeback at the end of the last century was stimulated by the necessity to study highly delocalized molecular excited states. In the SC method, a one-particle MO is represented with respect to a single center of the molecule via an expansion in terms of spherical harmonics. The traditional way of implementing this method is to search for partial harmonics of an SC 
expansion in form of a linear combination of Slater orbitals by minimization of the total energy of a molecular electronic state. ${ }^{27}$

In the case of a photoelectron in the continuum, it is convenient to utilize another version of the SC method, where the variational principle is applied to derive a system of coupled integrodifferential HF equations and these equations are solved numerically. ${ }^{28}$ This realization of the SC method was, at first, designed for the study of hydrogenous molecules ${ }^{28-32}$ and later on for the investigation of heavier nonhydrogenous molecules. ${ }^{33-36}$ In the last decade, an efficient scheme has been utilized for the numerical solution of a system of coupled HF equations, and the SC method has been successfully applied for the calculations of highly delocalized Rydberg states $^{37-40}$ and continuous partial photoelectron waves ${ }^{40-46}$ of simple diatomic molecules, such as $\mathrm{O}_{2}, \mathrm{~N}_{2}, \mathrm{NO}$, and $\mathrm{CO}$. It was demonstrated that the SC method allows for accurate calculations of total and partial cross sections and oscillator strengths, ${ }^{38-40}$ Auger decay rates, ${ }^{37,41,42}$ as well as angular distribution parameters for photoelectrons and fluorescence photons ${ }^{43-46}$ in molecules.

In order to attract more attention to the single center method, essentials of the method and its applications are presented in this paper. Basic equations of the method are given in Sec. II A. Efficient procedure for numerical solution of these equations and a practical scheme for noniterative accounting for exchange Coulomb interaction of the photoelectron with the core are described in Sec. II B. Several illustrative applications of the method to the theoretical description of photoexcitation of $\mathrm{O}_{2}$ (Sec. III A), resonant photoionization of $\mathrm{O}_{2}$ (Sec. III B), and angular distribution of photoelectrons and fluorescence photons in the core-excited $\mathrm{CO}$ and NO molecules (Sec. III C) are also shown. We conclude with a brief summary.

\section{THE SINGLE CENTER METHOD}

\section{A. Basic equations of the method}

According to the SC method, the spatial part of the MO is represented with respect to the single center of a molecule as an expansion by spherical harmonics $Y_{\ell m}(\theta, \varphi)$ with a given projection $m$ of the angular momentum $\ell$ on a chosen quantization axis $(z)$ :

$$
\Psi_{\varepsilon}(x, y, z)=\sum_{\ell m} \frac{P_{\varepsilon \ell m}(r)}{r} Y_{\ell m}(\theta, \varphi),
$$

where $x, y, z$ and $r, \theta, \varphi$ are the Cartesian and spherical coordinates with respect to the chosen center, $P_{\varepsilon \ell m}(r)$ stands for the radial parts of partial harmonics in the SC expansion of the MO. In the most general case, these radial parts are complex functions and summations in Eq. (1) over indices $\ell$ and $m$ are infinite. If, however, a particular symmetry of the MO is implied, summation over index $m$ is restricted according to this symmetry. For instance, in a diatomic molecule, where the projection $m$ on the molecular axis is a good quantum number, radial parts $P_{\varepsilon \ell m}(r)$ can be chosen real and summation over $m$ is omitted. Thereby, one defines $\sigma, \pi, \delta$ orbitals for $m=0, \pm 1, \pm 2$, respectively. There, the center of a diatomic molecule is practical to choose in the middle of the molecular axis ( $z$-axis) in between the two nuclei.

The radial parts $P_{\varepsilon \ell m}(r)$ of the photoelectron molecular orbital satisfy the following system of coupled Hartree-Fock equations: ${ }^{28,40}$

$$
\begin{aligned}
\sum_{\ell^{\prime} m^{\prime}}[ & \left(-\frac{1}{2} \frac{\mathrm{d}^{2}}{\mathrm{~d} r^{2}}+\frac{\ell(\ell+1)}{2 r^{2}}-\varepsilon\right) \delta_{\ell \ell^{\prime}} \delta_{m m^{\prime}} \\
& \left.+V_{\ell m \ell^{\prime} m^{\prime}}^{n e}(r)+V_{\ell m \ell^{\prime} m^{\prime}}^{e e}(r)\right] P_{\varepsilon \ell^{\prime} m^{\prime}}(r)=0,
\end{aligned}
$$

under the following normalization conditions for the discrete $P_{n \ell m}$ and continuous $P_{\varepsilon \ell m} \mathrm{MO}$ :

$$
\begin{aligned}
& \sum_{\ell m}\left\langle P_{n \ell m} \mid P_{n^{\prime} \ell m}\right\rangle=\delta_{n n^{\prime}}, \\
& \sum_{\ell m}\left\langle P_{\varepsilon \ell m} \mid P_{\varepsilon^{\prime} \ell m}\right\rangle=\delta\left(\varepsilon-\varepsilon^{\prime}\right) .
\end{aligned}
$$

In the system of equations (2) the following designations are used: $\varepsilon$ is the one-electron energy in atomic units, $V_{\ell m \ell^{\prime} m^{\prime}}^{n e}$ is the potential describing nuclear-electron interaction, and $V_{\ell m \ell^{\prime} m^{\prime}}^{e e}$ is the potential describing local direct $J_{\ell m \ell^{\prime} m^{\prime}}^{e e}$ and nonlocal exchange $K_{\ell m \ell^{\prime} m^{\prime}}^{e e}$ electrostatic Coulomb interactions of the photoelectron with the ionic core. Because of a nonspherical molecular field, the system of equations (2) contains the off-diagonal potentials with $\ell m \neq \ell^{\prime} m^{\prime}$, which couples equations for different partial harmonics. These potentials can be calculated as described below using the MO LCAO representation of the occupied shells deconvolved as Eq. (1).

Let $R_{n}, \theta_{n}, \phi_{n}$ are the spherical coordinates of the nucleus $n$ of charge $Z_{n}$ with respect to the chosen center. In these designations, the potential for the nuclei-electron interaction is given in atomic units as ${ }^{28}$

$$
\begin{aligned}
V_{\ell m \ell^{\prime} m^{\prime}}^{n e}= & -\sum_{n} Z_{n} \sum_{k}(-1)^{m} \sqrt{(2 \ell+1)\left(2 \ell^{\prime}+1\right)} \\
& \times\left(\begin{array}{ccc}
\ell & k & \ell^{\prime} \\
0 & 0 & 0
\end{array}\right)\left(\begin{array}{ccc}
\ell & k & \ell^{\prime} \\
-m & q & m^{\prime}
\end{array}\right) \\
& \times \sqrt{\frac{4 \pi}{2 k+1}} Y_{k q}^{*}\left(\theta_{n}, \phi_{n}\right) \frac{r_{<}^{k}}{r_{>}^{k+1}},
\end{aligned}
$$

where $r_{<}=\min \left(r, R_{n}\right), r_{>}=\max \left(r, R_{n}\right)$, and $q=m-m^{\prime}$. For a diatomic molecule aligned along the $z$-axis, index $q=0$ and the coefficient $\sqrt{4 \pi /(2 k+1)} Y_{k 0}^{*}\left(\theta_{n}, \phi_{n}\right)$ is equal to 1 or $(-1)^{k}$ for nuclei with the coordinates $(R, 0,0)$ or $(R, \pi, 0)$, respectively. It immediately implies that the potential (4) is diagonal over indices $m$ and $m^{\prime}\left(V_{\ell m \ell^{\prime} m^{\prime}}^{n e}=V_{\ell \ell^{\prime}}^{n e} \delta_{m m^{\prime}}\right)$ and $\sigma$, $\pi, \delta, \ldots$ orbitals do not mix. Moreover, for homonuclear diatomics only even values of index $k=2 i$ appear in Eq. (4), since for $k=2 i+1$ odd values $Z\left(1+(-1)^{2 i+1}\right)=0$. Thus, only partial harmonics with every second angular momentum $\ell$ are mixed by the nonspherical molecular field: even for gerade symmetry and odd for ungerade.

The potential describing direct Coulomb interaction of the photoelectron with electrons of the core can be computed as ${ }^{28}$ 


$$
\begin{aligned}
J_{\ell m \ell^{\prime} m^{\prime}}^{e e}= & \sum_{c} a_{c} \sum_{\ell_{c} m_{c}} \sum_{\ell_{c}^{\prime} m_{c}^{\prime}} \sum_{k}(-1)^{m_{c}+m^{\prime}} \\
& \times \sqrt{\left(2 \ell_{c}+1\right)\left(2 \ell_{c}^{\prime}+1\right)(2 \ell+1)\left(2 \ell^{\prime}+1\right)} \\
& \times\left(\begin{array}{ccc}
\ell_{c} & k & \ell_{c}^{\prime} \\
0 & 0 & 0
\end{array}\right)\left(\begin{array}{ccc}
\ell_{c} & k & \ell_{c}^{\prime} \\
-m_{c} & q & m_{c}^{\prime}
\end{array}\right)\left(\begin{array}{ccc}
\ell^{\prime} & k & \ell \\
0 & 0 & 0
\end{array}\right) \\
& \times\left(\begin{array}{ccc}
\ell^{\prime} & k & \ell \\
-m^{\prime} & q & m
\end{array}\right) y_{k}\left(\ell_{c} m_{c}, \ell_{c}^{\prime} m_{c}^{\prime}\right) .
\end{aligned}
$$

Index $c$ in Eq. (5) numerates the core electrons, indices $\ell_{c} m_{c}$ and $\ell_{c}^{\prime} m_{c}^{\prime}$ numerate partial harmonics of the bra- and ket- $\mathrm{MO}$ of the core, index $q$ is defined by $q=m_{c}-m_{c}^{\prime}=m^{\prime}-m$, and summation over index $k$ accounts for the partial harmonics of the electrostatic Coulomb potential defined in atomic units by

$$
y_{k}\left(\ell_{c} m_{c}, \ell_{c}^{\prime} m_{c}^{\prime}\right)=\int_{0}^{\infty} \frac{r_{<}^{k}}{r_{>}^{k+1}} P_{n_{c} \ell_{c} m_{c}}^{*}\left(r^{\prime}\right) P_{n_{c} \ell_{c}^{\prime} m_{c}^{\prime}}\left(r^{\prime}\right) \mathrm{d} r^{\prime},
$$

with $r_{>}=\max \left(r, r^{\prime}\right)$ and $r_{<}=\min \left(r, r^{\prime}\right)$. Nonlocal exchange Coulomb interaction of the photoelectron with the core can be computed similarly via

$$
\begin{aligned}
K_{\ell m \ell^{\prime} m^{\prime}}^{e e} P_{\varepsilon \ell^{\prime} m^{\prime}}= & \sum_{c} b_{c} \sum_{\ell_{c} m_{c}} \sum_{\ell_{c}^{\prime} m_{c}^{\prime}} \sum_{k}(-1)^{m_{c}+m_{c}^{\prime}} \\
& \times \sqrt{\left(2 \ell_{c}+1\right)\left(2 \ell_{c}^{\prime}+1\right)(2 \ell+1)\left(2 \ell^{\prime}+1\right)} \\
& \times\left(\begin{array}{ccc}
\ell_{c} & k & \ell^{\prime} \\
0 & 0 & 0
\end{array}\right)\left(\begin{array}{ccc}
\ell_{c} & k & \ell^{\prime} \\
-m_{c} & q & m^{\prime}
\end{array}\right) \\
& \times\left(\begin{array}{ccc}
\ell_{c}^{\prime} & k & \ell \\
0 & 0 & 0
\end{array}\right)\left(\begin{array}{ccc}
\ell_{c}^{\prime} & k & \ell \\
-m_{c}^{\prime} & q & m
\end{array}\right) \\
& \times y_{k}\left(\ell_{c} m_{c}, \ell^{\prime} m^{\prime}\right) P_{n_{c} \ell_{c}^{\prime} m_{c}^{\prime}} .
\end{aligned}
$$

Coefficients $a_{c}$ and $b_{c}$ in Eqs. (5) and (7) are determined by all quantum numbers of the electronic configuration formed by the ionic core and the photoelectron.

\section{B. Scheme for numerical solution}

The system of coupled differential equations of the second order [Eq. (2)] can be conveniently solved with the use of the Numerov finite-difference scheme, which relates the values of an unknown multicomponent function to three neighboring points. These equations can be numerically integrated using, for instance, asymptotic expansions of the desired functions in powers of $r^{\ell+k}(k=1,2, \ldots)$ at the origin of the coordinates. As a rule, system (2) couples the harmonics $P_{\varepsilon \ell m}$ over a wide range of angular momenta $\ell$. In the spatial range below the centrifugal barrier, the harmonics $P_{\varepsilon \ell m}$ corresponding to small and large values of the angular momentum $\ell$ differ from each other by several tens orders of magnitude. Consequently, this integration technique necessarily involves computational problems associated with the accuracy decrease. In order to overcome these difficulties, it is convenient to use the vector sweep method by reducing the Cauchy problem for the second-order equation to a boundary problem (see Appendix A). The main advantage of the method is that the sweep matrices are of the same order of magnitude over the entire range of angular momenta $\ell$. Such a combination of the Numerov finite-difference scheme and the sweep method is described in the monograph ${ }^{47}$ and was successfully applied to the calculations of atomic orbitals.

For the photoelectron in the discrete spectrum, the system of equations (2) relates solutions in the "closed channels." Integration of the system for the case of $\varepsilon<0$ must be performed in both directions. The nonlocal exchange Coulomb interaction of the photoelectron with the core electrons can be included, for instance, by an iterative procedure and calculated using the solutions $P_{\varepsilon \ell m}$ obtained in the preceding iterative step. In the first iteration step, the nonlocal term can be omitted. In this phase of the integration of the homogeneous system of coupled differential equations, matching of the desired functions and their derivatives obtained by integration in opposite directions results in the criteria for determination of the one-electron energy $\varepsilon$. When the exchange interaction is taken into account, the system of equations (2) has a unique solution for any one-electron energy $\varepsilon$. The invariability of the intermediate normalization of the desired function in the discrete spectrum can be used as a criterion to determine a unique value of the energy at each iteration step. After iterative procedures have converged, the molecular orbital must be normalized according to Eq. (3). Details of the integration of system of equations [Eq. (2)] in the discrete spectrum are summarized in Appendix B 1.

For the photoelectron in a continuous spectrum $(\varepsilon>0)$, the system of equations (2) relates solutions in the "open channels" and integration must be performed only outward. In the case of interaction of $C$ continuous spectra at a fixed energy, one obtains $C$ degenerate $C$-components solutions. According to Ref. 48, linearly independent solutions of the system of equations (2) at $r \rightarrow \infty$ should be sought in the following asymptotic form:

$$
P_{\ell m}^{L M}(r)=\delta_{\ell}^{L} \delta_{m}^{M} J_{\ell}(r)+\mathbb{R}_{\ell m}^{L M} H_{\ell}(r), \forall L M, \ell m=1, \ldots, C .
$$

Here, $J_{\ell}(r)$ and $H_{\ell}(r)$ are so-called Coulomb functions (the linearly independent and energy-normalized solutions in the spherically symmetric Coulomb potential). The superscripts $L M$ in Eq. (8) numerate the different degenerate multicomponent solutions, and the subscripts $\ell m$ number the different components of each solution. The complex hermitian $C \times C$ matrix $\mathbb{R}_{\ell m}^{L M}$ in Eq. (8) is known as the reaction matrix or $R$ matrix (see Appendix B 2 for details).

The new solutions, $\mathcal{P}_{\ell m}^{L M}(r)$, which are normalized on the energy scale according to Eq. (3), satisfy the condition of mutual orthogonality, and describe the partial photoelectron incoming-wave channels (outgoing spherical waves only in channel $\ell m=L M$ ), should be chosen as the following linear combinations of the solutions (8): $:^{49,50}$

$$
\begin{aligned}
\mathcal{P}_{\ell m}^{L M}= & \sum_{L^{\prime} M^{\prime}}\left\{\sum_{L^{\prime \prime} M^{\prime \prime}}\left(\widetilde{\mathbb{U}}_{L^{\prime} M^{\prime}}^{L^{\prime \prime} M^{\prime \prime}}\right)^{*}\right. \\
& \left.\times \cos \eta_{L^{\prime \prime} M^{\prime \prime}} e^{-i \eta_{L^{\prime \prime} M^{\prime \prime}}} \mathbb{U}_{L^{\prime \prime} M^{\prime \prime}}^{L M}\right\} P_{\ell m}^{L^{\prime} M^{\prime}},
\end{aligned}
$$


where complex vectors $\mathbb{U}_{L^{\prime} M^{\prime}}^{L M}$ are the solutions of the eigenvalue problem for the hermitian $R$-matrix with real eigenvalues $-\tan \eta_{L M}$ :

$$
\sum_{L^{\prime} M^{\prime}} \mathbb{R}_{\ell m}^{L^{\prime} M^{\prime}} \mathbb{U}_{L^{\prime} M^{\prime}}^{L M}=-\tan \eta_{L M} \mathbb{U}_{\ell m}^{L M} .
$$

We point out that after the transformation (9) partial continuum waves are always complex functions, even in the case of diatomics, where solutions (8) can be sought as real.

In order to increase the accuracy of the numerical procedure in the regions of singularity of molecular potentials, the system (A1) can be solved by changing the spatial integration variable $r$ with the new variable $\rho$ via the expression

$$
\rho(r)=\alpha r+\beta \ln r+\sum_{n} \arctan \frac{R_{n}-r}{\gamma_{n}} .
$$

Integration is performed in constant steps in the variable $\rho$. The second term in expression (11) makes it possible to concentrate the integration points at the center of the molecule, as suggested in Ref. 47. In addition, we have introduced the third term (sum runs over the nuclei centers) that symmetrically concentrates integration points around the positions of the nuclei $R_{n}$. It can be easily shown (see also Ref. 47) that replacing the solution functions $P_{\varepsilon \ell m}(r)$ by the new ones $F_{\varepsilon \ell m}(\rho)$ via

$$
P_{\varepsilon \ell m}=F_{\varepsilon \ell m}\left(\frac{\mathrm{d} \rho}{\mathrm{d} r}\right)^{-1 / 2}=\frac{F_{\varepsilon \ell m}}{\sqrt{ } \rho_{r}^{\prime}},
$$

results in the following system of coupled differential equations, which also do not contain the first derivative of the $F_{\varepsilon \ell m}(\rho)$ functions [compared with Eq. (A1)]:

$$
\begin{aligned}
\frac{\mathrm{d}^{2} F_{\varepsilon \ell m}(\rho)}{\mathrm{d} \rho^{2}}= & \frac{1}{\left(\rho_{r}^{\prime}\right)^{2}} \sum_{\ell^{\prime} m^{\prime}}\left[\left(\frac{\ell(\ell+1)}{r^{2}(\rho)}-2 \varepsilon\right) \delta_{\ell \ell^{\prime}} \delta_{m m^{\prime}}\right. \\
& +2 V_{\ell m \ell^{\prime} m^{\prime}}^{n e}(\rho)+2 J_{\ell m \ell^{\prime} m^{\prime}}^{e e}(\rho) \\
& \left.+\left\{\frac{1}{2} \frac{\rho_{r}^{\prime \prime \prime}}{\rho_{r}^{\prime}}-\frac{3}{4} \frac{\left(\rho_{r}^{\prime \prime}\right)^{2}}{\left(\rho_{r}^{\prime}\right)^{2}}\right\} \delta_{\ell \ell^{\prime}} \delta_{m m^{\prime}}\right] F_{\varepsilon \ell^{\prime} m^{\prime}}(\rho) \\
& +\frac{1}{\left(\rho_{r}^{\prime}\right)^{2}} \sum_{\ell^{\prime} m^{\prime}} 2 K_{\ell m \ell^{\prime} m^{\prime}}^{e e}(\rho) F_{\varepsilon \ell^{\prime} m^{\prime}}(\rho) .
\end{aligned}
$$

Finally, the solution of the system (2) becomes easier if one takes into account the persistency of the shape of $P_{\varepsilon \ell m}$ functions at high $\ell m$-values. This allows the restriction of the expansion (1) for simple diatomic molecules, such as $\mathrm{O}_{2}$, $\mathrm{N}_{2}$, NO, and CO, to $\ell \leq 20-25$, increasing the $V_{\ell m \ell^{\prime} m^{\prime}}^{n e}(r)$ and $V_{\ell m \ell^{\prime} m^{\prime}}^{e e}(r)$ potentials for large $\ell m .^{37-46}$ The inhomogeneous (due to exchange Coulomb interaction) system of coupled equations (2) can be solved, for instance, iteratively. ${ }^{40}$ The alternative approach implies that the system (2) and integral equations for exchange Coulomb potentials (6) can be reduced to a united homogeneous system of coupled differential equations relative to both partial harmonics and corresponding exchange potentials as suggested in Ref. 51 and described in details in Ref. 48. Essentials of this procedure are given in Appendix C.

\section{Computational details and application range}

Before discussing recent applications of the single center method, let us briefly outline relevant computational details, perspectives for going beyond the one-electron Hartree-Fock approximation, as well as its application range and limitations for heavy and polyatomic molecules.

The computational details will be outlined by two representative examples. The first is the calculation of the $\mathrm{CO}$ $5 \sigma^{-1} 3 \mathrm{~s} \sigma$ Rydberg orbital. We restrict the SC expansion (1) for the core MOs of $\mathrm{CO}$ and for the Rydberg electron to $\ell_{c} \leq 24$ and $\ell \leq 20$, respectively. The exchange Coulomb interaction of the Rydberg electron with the ionic core can be described by 45 generalized potentials (C5) for each of the core orbitals with the multiplicity $k$ running from 0 to 44 (except for the $1 \pi$ orbital where $k$ runs from 1 to 44$)$. Here, one has to solve 290 coupled differential equations (C8) for 269 exchange potentials and for 21 partial harmonics of the Rydberg electron $(\ell=0-20)$. The change of the integration variable according to Eq. (11) allows one to cover the radial interval of $0-35$ a.u. by only 250 grid points and, simultaneously, to obtain a relative accuracy in energy below $10^{-4}$. It becomes possible owing to increase of the accuracy of the numerical calculation in the regions of singularity of the molecular potential (concentration of the integration points at the center of the molecule and at the positions of the nuclei). This numerical problem required less than 0.5 GB memory and was solved by a contemporary single processor personal computer (PC) within 5 min.

The second example is the $3 \sigma_{g}^{-1} \varepsilon \sigma_{u}$ ionization of the $\mathrm{N}_{2}$ molecule. The following parameters were used in the calculations: $\ell_{c} \leq 30, \ell \leq 19$, and 350 grid points. As mentioned above, for homonuclear diatomic molecules every second angular momentum $\ell$ enters the SC expansion (1), and every second multiplicity $k$ of the generalized potentials (C5) is necessary to describe exchange interaction of the photoelectron with the ionic core. As a result one has to integrate only 159 coupled differential equations (C8): 149 equations for generalized exchange potentials and 10 equations for the partial harmonics of the continuum electron $(\ell=1,3, \ldots, 19)$. However, a complete solution requires integration of ten degenerate ten component continuous functions (8). This numerical problem required even less memory and was solved by a single processor PC within 2 min.

Of course, studying heavier molecules requires longer $\mathrm{SC}$ expansions over $\ell$, depending on the energy of an $\mathrm{MO}$ and on the internuclear distance. A maximal angular momentum in the expansion (1) grows with the one-electron energy $\varepsilon$ as $\sqrt{\varepsilon}$ and linearly with the coordinate $R$ of a nucleus with respect to the chosen center. Longer expansions over $\ell$, in turn, result in much longer expansions of the exchange potentials (C5) over multiplicity $k$. As is obvious from the considered examples, the latter quantities provide a major contribution to the size of the system of coupled differential equations (C8). A precise solution of this problem would require much more memory and a parallelization of the computer code. However, one can still solve this problem within a justified approximation. Major parts of storage memory and computational time can be reduced by restricting expansions of the generalized exchange 
potentials (C5) up to a certain value $k_{\max }$. The latter value can be estimated from the convergence of computational results with respect to the maximal multiplicity taken into account. The $k_{\max }$ value providing satisfactory results ranges usually from 30 to 50, depending on the molecule. Applications of the method to heavy molecules can be found in Refs. 33-36.

In the case of nonlinear polyatomic molecules, the SC representation of an MO includes an additional expansion over index $m$. However, the largest value of the projection of the angular momentum in the expansion (1) must not necessarily coincide with the largest angular momentum. Depending on the geometry of the molecule it can be chosen smaller. In addition, summation over index $m$ in the expansion (1) is always restricted according to the symmetry of an MO. Most importantly, expansion over $m$ does not increase the number of the generalized potentials (C5), since all summations over indices $m$ and $m_{c}$ in Eqs. (C10) are performed analytically (multiplicity $k$ of potentials (C5) depends solely on $\ell$ and $\ell_{c}$ values). These facts allow the straightforward application of the method to nonlinear polyatomic molecules, as has been done for $\mathrm{H}_{2} \mathrm{O}, \mathrm{H}_{2} \mathrm{~S}, \mathrm{NH}_{3}, \mathrm{PH}_{3}, \mathrm{CH}_{4}$, and $\mathrm{SiH}_{4}$ in Refs. 28-32.

One of the most important advantages of the single center method is the "atomiclike" representation of the molecular orbitals. The representation of an MO via Eq. (1) allows the straightforward implementation of numerous existing methods elaborated for inclusion of many-electron correlations in atoms. Among them are configuration interaction expansions, multireference approaches, many-body perturbation theory, and random-phase-approximation. For instance, the effect of many-electron correlations on valence and subvalence shells ionization of molecules has been studied by the SC method in Refs. 28-32. There, the effect of the polarization of valence shells by a subvalence shell vacancy has been described by a two-reference representation of a final ionic state, and the random-phase-approximation has been implemented in order to include intershell and intrashell correlations. In the recent studies of core-excited molecules, ${ }^{43-46}$ the monopole relaxation of a molecular core was taken into account within the sudden approximation by applying the theory of nonorthogonal orbitals.

\section{APPLICATIONS}

\section{A. Discrete spectrum: Highly excited molecular Rydberg states}

In this subsection we discuss applications of the SC method to the calculation of MOs of a photoelectron in the discrete spectrum. In Refs. 37 and 40 properties of the highly delocalized $\mathrm{O}_{2}^{*}\left(c^{4} \Sigma_{u}^{-}\right) n \ell \sigma_{g}{ }^{3} \Sigma_{u}^{-}$molecular Rydberg states have been computed. This allowed a quantitative interpretation of the experimentally observed competition between the neutral predissociation and autoionization of these resonant states (see Sec. III B). Recently, ${ }^{38}$ the SC method has been successfully applied to the interpretation of a predominant dissociation of the $\mathrm{CO}^{*}\left(\mathrm{D}^{2} \Pi\right) n(\mathrm{~d} / \mathrm{s}) \sigma^{1} \Pi$ Rydberg states into atomic excited Rydberg fragments with the same effective principal quantum number. Below we briefly discuss very recent results from Ref. 39, where the competi-
TABLE I. Properties of some $\mathrm{O}_{2}^{*}\left(a^{4} \Pi_{u}\right) n \ell \sigma_{g} / n \ell \delta_{g}{ }^{3} \Pi_{u}$ molecular Rydberg states computed by the SC method in Ref. 39 at the internuclear distance $R=2.28$ a.u.

\begin{tabular}{|c|c|c|c|c|c|c|c|}
\hline & \multicolumn{2}{|c|}{ Assignment } & \multicolumn{2}{|c|}{$I P / \varepsilon^{\mathrm{a}}(\mathrm{eV})$} & \multirow{2}{*}{$\begin{array}{c}\bar{r}^{\mathrm{b}} \text { (a.u.) } \\
\mathrm{SC}\end{array}$} & \multicolumn{2}{|c|}{$\langle\ell m \mid \ell m\rangle^{\mathrm{c}}(\%)$} \\
\hline & Ref. 53 & Ref. 54/SC & Ref. 53 & SC & & $\mathrm{s}$ & d \\
\hline$\overline{\mathrm{H}}$ & $3 \mathrm{~s} \sigma_{g}$ & $3 \mathbf{s} \sigma_{g}$ & 3.727 & 3.591 & 5.74 & 93.6 & 6.1 \\
\hline I & $3 \mathrm{~d} \sigma_{g}$ & $4 s \sigma_{g}$ & 1.690 & 1.649 & 11.3 & 67.7 & 32.2 \\
\hline $\mathrm{I}^{\prime}$ & $4 \mathrm{~s} \sigma_{g}$ & $\mathbf{3 d} \delta_{g}$ & 1.521 & 1.520 & 10.4 & 0.1 & 99.9 \\
\hline $\mathrm{I}^{\prime \prime}$ & $3 \mathrm{~d} \delta_{g}$ & $\mathbf{3 d} \sigma_{g}$ & 1.488 & 1.456 & 12.4 & 31.4 & 68.5 \\
\hline
\end{tabular}

${ }^{a}$ Experimental ionization potential (theoretical single-electron energy) of the MO.

${ }^{\mathrm{b}}$ Average radius of the MO.

${ }^{\mathrm{c}}$ Norm of the partial harmonic $P_{n \ell m}$ in percent. The prevailing integrals are indicated in bold.

tion between autoionization and neutral dissociation of the I, I', and I" vibronic Rydberg processions of $\mathrm{O}_{2}$ has been studied. There, predissociation of these molecular Rydberg states results in the fragmentation of a molecule into $\mathrm{O}\left(2 \mathrm{p}^{4}{ }^{3} \mathrm{P}_{J}\right)+\mathrm{O}^{*}\left(2 \mathrm{p}^{3}\left({ }^{4} \mathrm{~S}\right) 3 \mathrm{~s}^{3} \mathrm{~S}_{1}\right)$ fragments, and the excited atomic fragment decays further via emission of fluorescence photon. Computed electronic properties of the I, $\mathrm{I}^{\prime}$, and $\mathrm{I}^{\prime \prime}$ states allowed the interpretation of the experimental atomic fluorescence spectra from Ref. 52.

Some properties of the $\mathrm{O}_{2}^{*}\left(a^{4} \Pi_{u}\right) n \ell \sigma_{g} / n \ell \delta_{g}{ }^{3} \Pi_{u}$ Rydberg states calculated by the SC method in Ref. 39 are collected in Table I. One can see from this table that the experimental ionization potentials from Ref. 53 can be satisfactorily reproduced by the SC one-electron energies of Rydberg electrons (cf. columns 4 and 5), even for delocalized electrons (average radii are listed in column 6). Columns 7 and 8 show the norms of the partial harmonics $\langle\ell m \mid \ell m\rangle$, which characterize the part of the MO described by the given angular momentum. The main contributions used for the assignment of the Rydberg electrons in column 3 are set in bold. The first Rydberg state is described mainly by s symmetry and is separated from the next group of states by an energy gap. In the next group, a practically pure d state of $\delta_{g}$ symmetry appears in between two $\sigma_{g}$ states, which are strongly mixed with each other. The strong $n \mathrm{~s} /(n-1) \mathrm{d}$ mixing for the energetically close Rydberg states of $\sigma$ symmetry was found to be a general effect in small homonuclear ${ }^{37,40}$ and almost symmetric heteronuclear ${ }^{38}$ molecules. The first $\sigma_{g}$ state has the largest admixture of the s symmetry and the second one that of the $\mathrm{d}$ symmetry. These facts allowed the reassignment of the I, $\mathrm{I}^{\prime}$, and $\mathrm{I}^{\prime \prime}$ states as given in column 3. Although the reassignment suggested in Ref. 39 was in contradiction to the assignment of Ref. 53 based on a quantum defect analysis (column 2 of Table I), it supports the later reassignment ${ }^{54}$ based on the analysis of the autoionization dynamics of the spin-orbit components of these states.

\section{B. Continuous spectrum: Photoionization via autoionization of resonances}

Recently, ${ }^{41-46}$ the SC method has been applied to computations of absolute partial and total Auger (autoionization) decay rates of the core-ionized (core-excited) $\mathrm{N}_{2}$, $\mathrm{CO}$, and NO molecules. The theoretical decay rates were found in a good agreement with the available theoretical ${ }^{19-21}$ and experimental ${ }^{55-57}$ data. Computed absolute oscillator 


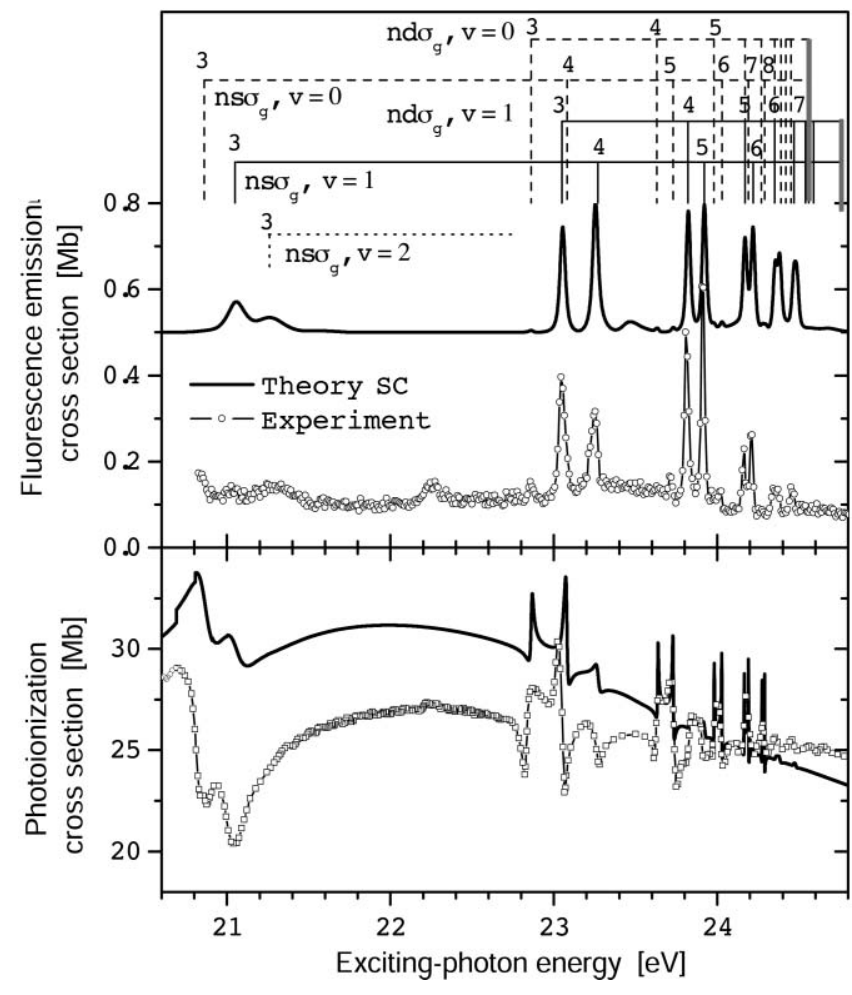

FIG. 1. Upper panel: Total cross sections for emission of atomic fluorescence in the range of $97-131 \mathrm{~nm}$ after predissociative photoionization of the oxygen molecule in the exciting-photon energy range of 20.6-24.8 eV computed by the SC method in Ref. 40 and measured in Ref. 58. The solid curve is shifted by $+0.5 \mathrm{Mb}$. Lower panel: Total photoionization cross sections computed by SC method in Ref. 40 and measured in Ref. 59. The theoretical cross sections are broadened by the instrumental Gaussian function of $20 \mathrm{meV}$ FWHM. Experimental positions of the $\mathrm{O}_{2}^{*}\left(c^{4} \Sigma_{u}^{-}\right) n \ell \sigma_{g}{ }^{3} \Sigma_{u}^{-}, v$ vibronic resonances from Ref. 53 are shown on the top.

strengths for core-excitations of these molecules and absolute partial and total autoionization rates allowed a quantitative interpretation of their experimental core-excitation spectra (see Sec. III C). In this subsection, we briefly discuss results of the calculation of the resonant photoabsorption cross sections of $\mathrm{O}_{2}$ in the exciting-photon energy range of $20.6-24.8 \mathrm{eV}$, obtained in Refs. 37 and 40. In this energy range, absorption of a photon results in ionization of the $3 \sigma_{g}, 1 \pi_{u}, 1 \pi_{g}$ valence shells of $\mathrm{O}_{2}$ or in the excitation of the $2 \sigma_{u}^{-1}\left(c^{4} \Sigma_{u}^{-}\right) n \sigma_{g} \mathrm{Ry}-$ dberg states. In the next step, these excited states can either autoionize into the mentioned ionization continua or can predissociate into the $\mathrm{O}\left(2 \mathrm{p}^{4}{ }^{1} \mathrm{D}\right)+\mathrm{O}^{*}\left(2 \mathrm{p}^{3}\left({ }^{4} \mathrm{~S}\right) n(\mathrm{~d} / \mathrm{s}){ }^{3} \mathrm{~S}\right)$ limits. In the latter case, excited atomic fragments relax further via emission of a fluorescence photon in the range of 97-131 $\mathrm{nm}$. Competition between the autoionization and predissociation pathways of the excited vibronic $2 \sigma_{u}^{-1}\left(c^{4} \Sigma_{u}^{-}\right) n \sigma_{g}, v$ Rydberg states of $\mathrm{O}_{2}$ results in the resonances of different shapes in the cross sections for photoionization and for atomic fluorescence emission (proportional to cross section for neutral dissociation ${ }^{58}$ ).

The absolute total photoionization cross section of $\mathrm{O}_{2}$ and the corresponding cross section for fluorescence emission computed in Ref. 40 in the exciting-photon energy range of 20.6-24.8 eV are compared in Fig. 1 with the corresponding experimental data from Refs. 58 and 59. Experimental positions of the $\mathrm{O}_{2}^{*}\left(c^{4} \Sigma_{u}^{-}\right) n \ell \sigma_{g}{ }^{3} \Sigma_{u}^{-}, v$ vi- bronic resonances are indicated at the top of the figure. In the calculations, ${ }^{40}$ it was assumed that the predissociation of the molecular $2 \sigma_{u}^{-1}\left(c^{4} \Sigma_{u}^{-}\right) n \sigma_{g}, v$ Rydberg states takes place mainly due to the predissociation of the molecular ionic core $2 \sigma_{u}^{-1}\left(c^{4} \Sigma_{u}^{-}\right), v$, and the delocalized Rydberg electrons are spectators of the process. Analysis of the computed absolute predissociation and autoionization rates yielded that (i) the predissociation width of the vibrational state $v=0$ is considerably smaller than the autoionization widths of Rydberg electrons and (ii) for the vibrational state $v=1$ these widths are comparable to each other. As a result, the $2 \sigma_{u}^{-1}\left(c^{4} \Sigma_{u}^{-}\right) n \sigma_{g}, v=0$ resonances manifest themselves mainly in the photoionization cross section (lower panel of Fig. 1), whereas the $2 \sigma_{u}^{-1}\left(c^{4} \Sigma_{u}^{-}\right) n \sigma_{g}, v=1$ resonances are observed in both the photoionization and fluorescence emission cross sections (lower and upper panels of Fig. 1). The figure illustrates good overall agreement between the resonance's shapes in the theoretical and experimental cross sections.

\section{Continuous spectrum: Angularly resolved deexcitation spectra of molecules}

The aim of this subsection is to demonstrate the potential of the SC method for computing angularly resolved decay spectra of molecules. Here, a precise theoretical description of the partial photoelectron waves $\ell m$ in the molecular continuum is necessary. The angularly resolved Auger electron and subsequent fluorescence emission spectra of the core-excited CO (Refs. 43 and 44) and NO (Refs. 45 and 46) molecules are discussed below.

Vibrationally and angularly resolved $\mathrm{CO}^{+} A^{2} \Pi$ decay spectra of the $\mathrm{C}^{*} \mathrm{O}$ and $\mathrm{CO}^{*}$ resonances were studied theoretically in Refs. 43 and 44, respectively. In Fig. 2, the partial photoionization cross section (panel a) and photoelectron angular distribution parameter (panel b) for the $\mathrm{CO}^{+} A^{2} \Pi\left(v^{\prime}=0\right)$ vibronic state as well as the angular distribution parameter for the subsequent $A^{2} \Pi\left(v^{\prime}=0\right) \rightarrow$ $X^{2} \Sigma^{+}\left(v^{\prime \prime}\right)$ fluorescence (panel c), computed in Ref. 43 by the SC method, are compared with the corresponding experimental data measured in Refs. 43 and 60. The figure illustrates a good quantitative agreement between the computed and measured angular distribution parameters $\beta^{e}$ and $\beta 2^{f l}$, supporting the high quality of photoelectron partial waves computed by the SC method. The $\mathrm{C}\left(1 \mathrm{~s} \rightarrow \pi^{*}\right)$ core excitation of $\mathrm{CO}$ satisfies the condition for weak lifetime vibrational interference (LVI) (Ref. 61): vibrational energy spacing between the $v_{r}$ levels of the $\mathrm{C}^{*} \mathrm{O}$ resonance, $\omega_{e}=250 \mathrm{meV}$ (Ref. 21), is about three times larger than their natural lifetime width, $\Gamma_{\pi^{*}}=80 \mathrm{meV} .^{56}$ Therefore, the LVI is practically invisible in the magic-angle-recorded decay spectra of this resonance. ${ }^{21,60,62}$ However, in the angularly resolved decay spectra (corresponding angular distribution parameters), the effect of LVI is more "visible." As one can see from panels (b) and (c) of Fig. 2, the measured angular distribution parameters $\beta^{e}$ and $\beta 2^{f l}$ exhibit substantial variation across the positions of the vibrational levels $v_{r}$ of the $\mathrm{C}^{*} \mathrm{O}$ resonance. Calculations performed in Ref. 43 support these observations and assign these variations to the LVI effect. 


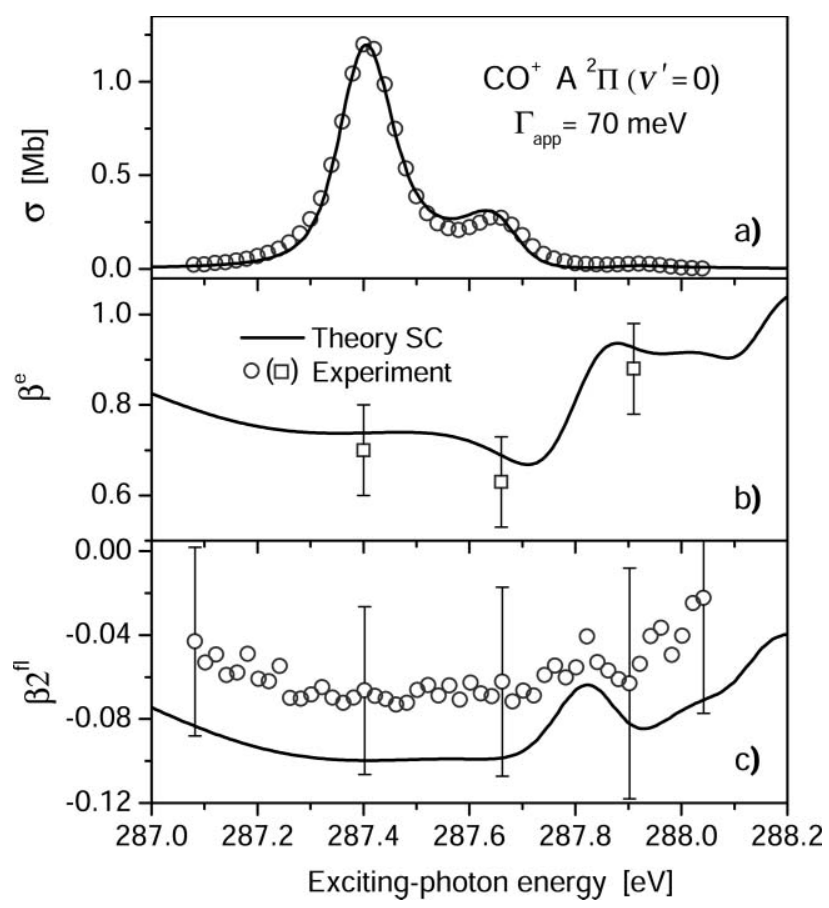

FIG. 2. Panel (a): Cross section for the population of the $\mathrm{CO}^{+} A^{2} \Pi\left(v^{\prime}=0\right)$ vibronic state in the vicinity of the $\mathrm{C}^{*} \mathrm{O}$ resonance. Theory and experiment from Ref. 43. Panel (b): Angular distribution parameter for the $\mathrm{CO}^{+} A^{2} \Pi\left(v^{\prime}=0\right)$ photoelectrons. Theory from Ref. 43 and experiment from Ref. 60. Panel (c): Angular distribution parameter for the $A^{2} \Pi\left(v^{\prime}=0\right) \rightarrow X^{2} \Sigma^{+}\left(v^{\prime \prime}\right)$ fluorescence bands progression. Theory and experiment from Ref. 43. The computed parameters were additionally convolved with a Gaussian of $70 \mathrm{meV}$ FWHM.

The angularly resolved Auger decay spectra of the coreexcited NO molecule have been measured in Ref. 63. There, a large disagreement between the measured $\mathrm{NO}^{+}\left(X^{1} \Sigma^{+}\right)$ Auger electron angular distribution parameters and theoretical parameters computed within the "standard LVI" theory in Ref. 63 have been found and tentatively assigned to the interference between overlapping electronic states within the multiplet structure of the resonance (known as electronic state interference (ESI) (Ref. 64). The effect of ESI on the angularly resolved decay spectra of the core-excited NO molecule, which is symmetry forbidden in the solid-angle-averaged (magic-angle-recorded) spectra, has recently been verified in Refs. 45 and 46. Experimental ${ }^{63}$ and theoretical ${ }^{46}$ parameters $\beta^{e}$ for the $\mathrm{NO}^{+}\left(X^{1} \Sigma^{+}\right)$Auger electrons are compared in the upper and lower panels of Fig. 3 as functions of the vibrational quantum number $v^{\prime \prime}$ at different exciting-photon energies (indicated in the figure). Good quantitative agreement between the theory and experiment supports the effect of interference between amplitudes for population and decay of different by symmetry intermediate resonances $1 \mathrm{~s}^{-1} 2 \pi^{2}\left({ }^{2} \Delta,{ }^{2} \Sigma^{ \pm}\right)$, as suggested in Ref. 63.

The angularly resolved $A^{1} \Pi \rightarrow X^{1} \Sigma^{+}$fluorescence spectra of the resonantly populated $\mathrm{NO}^{+}$ion at the $\mathrm{N}(1 \mathrm{~s}$ $\left.\rightarrow \pi^{*}\right)$ and $\mathrm{O}\left(1 \mathrm{~s} \rightarrow \pi^{*}\right)$ excitations of $\mathrm{NO}$ were studied in Refs. 45 and 46. The theoretical photoionization cross section of the $\mathrm{NO}^{+} A^{1} \Pi\left(v^{\prime}=0\right)$ state and angular distribution parameter for the $A^{1} \Pi\left(v^{\prime}=0\right) \rightarrow X^{1} \Sigma^{+}\left(v^{\prime \prime}\right)$ fluorescence, computed by the SC method in the vicinity of the $\mathrm{N}^{*} \mathrm{O}$ resonance, are compared with the corresponding experimental

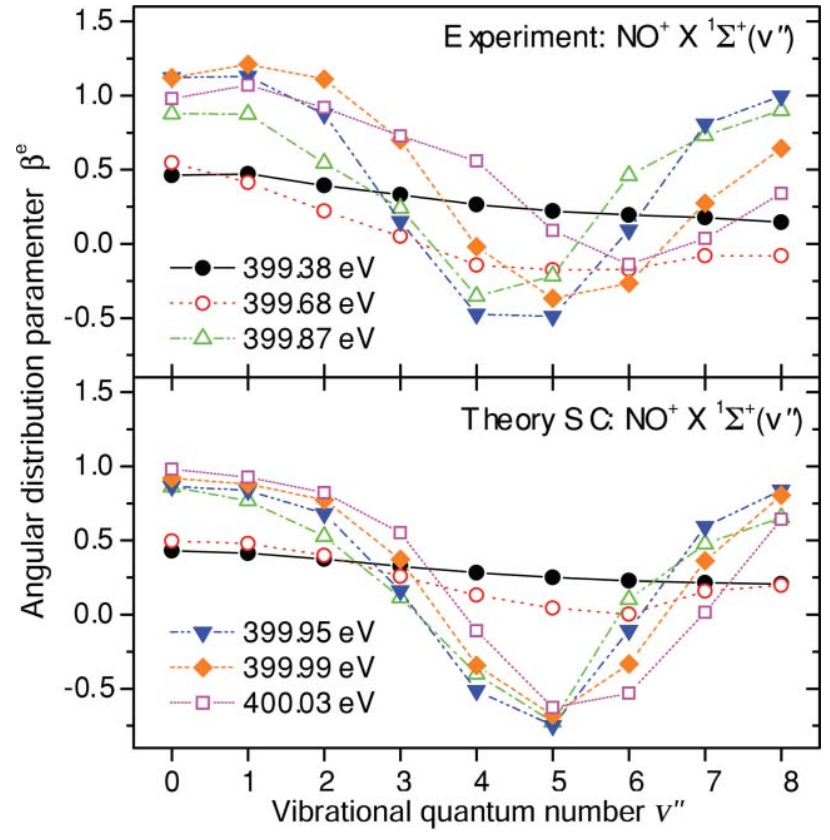

FIG. 3. Angular distribution parameters for the $\mathrm{NO}^{+} X^{1} \Sigma^{+}\left(v^{\prime \prime}\right)$ photoelectrons measured in Ref. 63 (upper panel) and computed by the SC method in Ref. 46 (lower panel) as functions of the vibrational quantum number $v^{\prime \prime}$ for selected exciting-photon energies in the vicinity of the $\mathrm{N}^{*} \mathrm{O}$ resonance.

data in Fig. 4. Here, the interference results in strong excitingphoton energy dependence of the angular distribution parameter across the positions of electronic resonances and is responsible for the change of sign of the $\beta 2^{f l}(\omega)$ function twice, as also observed in the experiment (panel (b) of Fig. 4). Good quantitative agreement between the computed and measured parameters $\beta 2^{f l}$ indicates the quality of partial

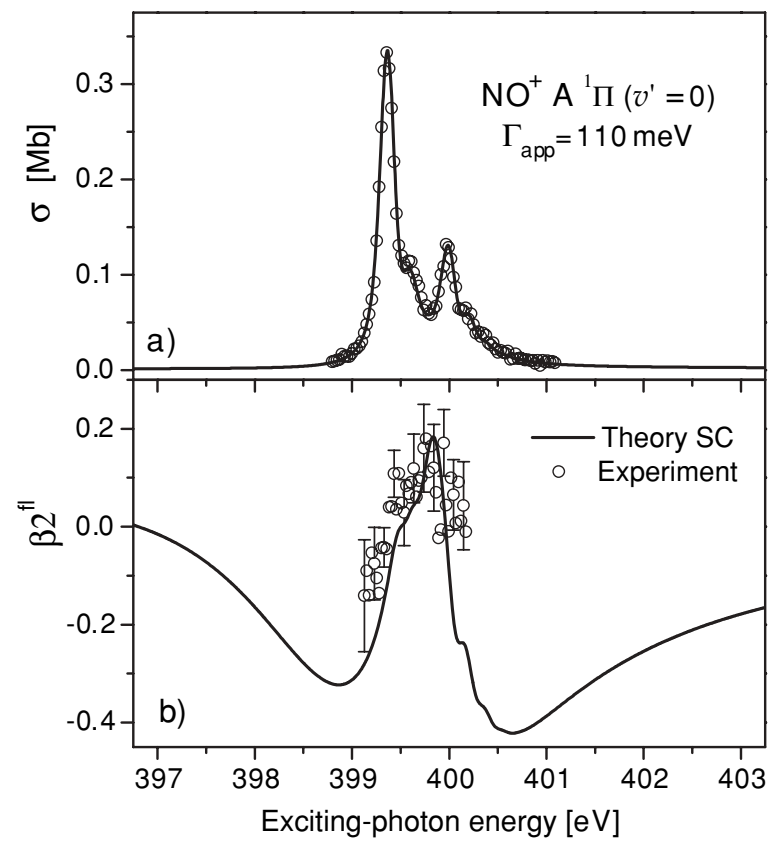

FIG. 4. Theory and experiment from Ref. 45. Panel (a): Cross section for the population of the $\mathrm{NO}^{+} A^{1} \Pi\left(v^{\prime}=0\right)$ vibronic state across the $\mathrm{N}^{*} \mathrm{O}$ resonance. Panel (b): Angular distribution parameter for the $A^{1} \Pi\left(v^{\prime}=0\right)$ $\rightarrow X^{1} \Sigma^{+}\left(v^{\prime \prime}\right)$ fluorescence bands progression. Computed parameters were convolved with a Gaussian of 110 meV FWHM. 
continuum photoelectron waves computed by the SC method and supports the effect of ESI on the angularly resolved fluorescence spectra suggested by the theory. ${ }^{45,46}$

\section{SUMMARY}

Basic equations of the SC method for the calculation of highly delocalized discrete and partial photoelectron continuum functions of molecules are presented, and an efficient scheme for the numerical solution is discussed. The method implies representation of MO as an expansion over spherical harmonics with respect to a chosen single center and subsequent solution of a system of coupled Hartree-Fock differential equations for a photoelectron in discrete or continuous spectra. The scheme implies the combination of the $\mathrm{Nu}-$ merov finite-difference scheme with the vector sweep method and allows noniterative inclusion of nonlocal exchange interaction of a photoelectron with a molecular core. All necessary relations for the numerical procedure are provided in the Appendices. Implementation of the method results in an accurate and stable numerical tool for the theoretical study of angularly resolved molecular photoabsorption problems. By several illustrative applications of the method to diatomic molecules, we reveal its potential for studying molecular photoionization in the discrete and continuous spectra. The method allows straightforward application to nonlinear polyatomic molecules. We hope this short overview will attract more attention to the method.

\section{ACKNOWLEDGMENTS}

The authors would like to thank B. M. Lagutin and I. D. Petrov for many valuable discussions. Financial support from the Deutsche Forschungsgemeinschaft (DFG) is gratefully acknowledged.

\section{APPENDIX A: DETAILS OF THE NUMERICAL PROCEDURE}

For the sake of convenience the system of equations (2) can be rewritten as

$$
\begin{aligned}
\frac{\mathrm{d}^{2} P_{\varepsilon \ell m}(r)}{\mathrm{d} r^{2}}= & \sum_{\ell^{\prime} m^{\prime}}\left[\left(\frac{\ell(\ell+1)}{r^{2}}-2 \varepsilon\right) \delta_{\ell \ell^{\prime}} \delta_{m m^{\prime}}\right. \\
& \left.+2 V_{\ell m \ell^{\prime} m^{\prime}}^{n e}(r)+2 J_{\ell m \ell^{\prime} m^{\prime}}^{e e}(r)\right] P_{\varepsilon \ell^{\prime} m^{\prime}}(r) \\
& +\sum_{\ell^{\prime} m^{\prime}} 2 K_{\ell m \ell^{\prime} m^{\prime}}^{e e}(r) P_{\varepsilon \ell^{\prime} m^{\prime}}(r) .
\end{aligned}
$$

By introducing the solution vector $\bar{P}=\left\{P_{\ell m}\right\}$, the nonlocal exchange vector $\bar{X}=\left\{X_{\ell m}\right\}=\sum_{\ell^{\prime} m^{\prime}} 2 K_{\ell m \ell^{\prime} m^{\prime}}^{e e}(r) P_{\varepsilon \ell^{\prime} m^{\prime}}$, and the matrix $\hat{F}=\left\{F_{\ell m \ell^{\prime} m^{\prime}}\right\}=\left(\ell(\ell+1) / r^{2}-2 \varepsilon\right) \delta_{\ell \ell^{\prime}} \delta_{m m^{\prime}}$ $+2 V_{\ell m \ell^{\prime} m^{\prime}}^{n e}(r)+2 J_{\ell m \ell^{\prime} m^{\prime}}^{e e}(r)$, the system (A1) takes the following matrix form:

$$
\frac{\mathrm{d}^{2} \bar{P}}{\mathrm{~d} r^{2}}=\hat{F} \times \bar{P}+\bar{X} .
$$

The Numerov finite-difference scheme relates the values of an unknown multicomponent function satisfying Eq. (A2), which do not include the first derivative of the desired function, to three neighboring points as

$$
\hat{a}_{n+1} \times \bar{P}_{n+1}-\hat{b}_{n} \times \bar{P}_{n}+\hat{a}_{n-1} \times \bar{P}_{n-1}=\overline{f_{n}}+O\left(h^{6}\right),
$$

with the $\hat{a}, \hat{b}$, and $\bar{f}$ matrices defined by

$$
\begin{aligned}
& \hat{a}_{n}=\left[\hat{E}-\frac{h^{2}}{12} \hat{F}_{n}\right], \quad \hat{b}_{n}=\left[2 \hat{E}+\frac{10 h^{2}}{12} \hat{F}_{n}\right], \\
& \overline{f_{n}}=\frac{h^{2}}{12}\left[\bar{X}_{n+1}+10 \bar{X}_{n}+\bar{X}_{n-1}\right] .
\end{aligned}
$$

Here, $\hat{E}$ is the unity matrix and subscript $n$ numerates grid points with a constant integration step $h$.

The outward (out) or inward (in) vector sweep relates the values of the unknown multicomponent function $\bar{P}$ at only two neighboring points $n$ and $n+1$ or $n-1$ and $n$, respectively, via

$$
\begin{gathered}
\bar{P}_{n}^{\text {out }}=\bar{U}_{n}^{\text {out }}+\hat{V}_{n}^{\text {out }} \times \bar{P}_{n+1}^{\text {out }}, \\
\bar{P}_{n}^{\text {in }}=\bar{U}_{n}^{\text {in }}+\hat{V}_{n}^{\text {in }} \times \bar{P}_{n-1}^{\text {in }} .
\end{gathered}
$$

Substitution of expressions (A5) into Eq. (A3) allows determination of recurrent matrix relations for the $\hat{V}_{n}$ and $\bar{U}_{n}$ matrices. Using the boundary condition for exponential decrease of the desired multicomponent function in the spatial range below the potential barrier, it is possible to reconstruct these matrices at the whole coordinate grid. For the case of the outward vector sweep, these relations read as

$$
\begin{aligned}
& \hat{V}_{n}^{\text {out }}=\left(\hat{b}_{n}-\hat{a}_{n-1} \times \hat{V}_{n-1}^{\text {out }}\right)^{-1} \times \hat{a}_{n+1}, \\
& \bar{U}_{n}^{\text {out }}=\left(\hat{b}_{n}-\hat{a}_{n-1} \times \hat{V}_{n-1}^{\text {out }}\right)^{-1} \times\left(\hat{a}_{n-1} \times \bar{U}_{n-1}^{\text {out }}-\bar{f}_{n}\right) .
\end{aligned}
$$

The boundary conditions for sweep matrices at $r \rightarrow 0$,

$$
\hat{V}_{1}^{\text {out }}=\hat{V}_{2}^{\text {out }}=\hat{V}, \quad \bar{U}_{1}^{\text {out }}=\bar{U}_{2}^{\text {out }}=0, \bar{f}_{1}=\bar{f}_{2}=0,
$$

allow one to start the outward vector sweep by solving the following equation relatively to the unknown matrix $\hat{V}$ :

$$
\hat{V}=\left(\hat{b}_{2}-\hat{a}_{1} \times \hat{V}\right)^{-1} \times \hat{a}_{3} .
$$

The inward vector sweep can only be applied to solutions in the discrete spectrum $(\varepsilon<0)$. Similar relations for matrices $\hat{V}_{n}^{\text {in }}$ and $\bar{U}_{n}^{\text {in }}$ read as

$$
\begin{aligned}
& \hat{V}_{n}^{\text {in }}=\left(\hat{b}_{n}-\hat{a}_{n+1} \times \hat{V}_{n+1}^{\text {in }}\right)^{-1} \times \hat{a}_{n-1}, \\
& \bar{U}_{n}^{\text {in }}=\left(\hat{b}_{n}-\hat{a}_{n+1} \times \hat{V}_{n+1}^{\text {in }}\right)^{-1} \times\left(\hat{a}_{n+1} \times \bar{U}_{n+1}^{\text {in }}-\bar{f}_{n}\right) .
\end{aligned}
$$

Integration of Eqs. (A9) can be started by applying the boundary conditions at $r \rightarrow \infty$,

$$
\hat{V}_{N}^{\text {in }}=\hat{V}_{N-1}^{\text {in }}=\hat{V}, \quad \bar{U}_{N}^{\text {in }}=\bar{U}_{N-1}^{\text {in }}=0, \quad \bar{f}_{N}=\bar{f}_{N-1}=0,
$$


resulting in the following equation relatively to the unknown matrix $\hat{V}$ :

$$
\hat{V}=\left(\hat{b}_{N-1}-\hat{a}_{N} \times \hat{V}\right)^{-1} \times \hat{a}_{N-2} .
$$

\section{APPENDIX B: DETAILS OF THE INTEGRATION}

\section{Discrete spectrum}

Equations (A6)-(A8) and (A9)-(A11) allow one to determine sweep matrices $\hat{V}_{n}^{\text {out }}, \bar{U}_{n}^{\text {out }}, n=1, \ldots, K+1$, and $\hat{V}_{n}^{\text {in }}, \bar{U}_{n}^{\text {in }}, n=K+1, \ldots, N$, respectively, at each trial energy $\varepsilon$. Matching the solutions of Eq. (A2), integrated in the two opposite directions, and their derivations at the grid point $K$ results in the following system of linear equations relative to the values $\bar{P}_{K}$ of the desired vector function in this point:

$$
\left(\left[\hat{V}_{K}^{\text {out }}\right]^{-1}-\hat{V}_{K+1}^{\text {in }}\right) \times \bar{P}_{K}=\bar{U}_{K+1}^{\text {in }}+\left[\hat{V}_{K}^{\text {out }}\right]^{-1} \times \bar{U}_{K}^{\text {out }} .
$$

In absence of the exchange Coulomb potentials (i.e., $\bar{X}=0$ ), the right hand side of Eq. (B1) is equal to zero. The homogeneous system of linear equations (B1) has a nontrivial solution if

$$
\operatorname{det}\left(\left[\hat{V}_{K}^{\text {out }}\right]^{-1}-\hat{V}_{K+1}^{\text {in }}\right)=0
$$

The latter equations are the criteria to determine the oneelectron energy $\varepsilon$ for the solution of the homogeneous system of equations (A2). Assuming that the solution $\left(P_{\ell_{1} m_{1}}\right)_{K}$ in the first channel $\ell_{1} m_{1}$ has the value of 1 in the matching point $K$, it is possible to solve Eq. (B1) relative to values of the other components $\ell m$ at point $K$ and to reconstruct the solution $\bar{P}$ in the whole coordinate grid via vector sweep relations (A5). It is obvious that if exchange Coulomb interaction is taken into account (i.e., $\bar{X} \neq 0$ ), the system of linear equations (B1) has a unique solution for any one-electron energy $\varepsilon$. In this case, the invariability of the intermediate normalization of the desired discrete function,

$$
\sum_{\ell m}\left\langle P_{n \ell m} \mid P_{n \ell m}\right\rangle=\text { const. }
$$

is the criterion to determine a unique value of the energy at each iteration step.

\section{Continuum spectrum}

Let the vector solution $\bar{P}^{L M}$ in the channel $L M$ has unknown values $\left(P_{\ell m}^{L M}\right)_{N}$ in the last grid point $N$. Using the vector sweep relations (A5) and the asymptotical condition (8) in the last two points of the integration grid $N$ and $N-1$, one can write

$$
\left\{\begin{array}{l}
\left(P_{\ell m}^{L M}\right)_{N}-\mathbb{R}_{\ell m}^{L M}\left(H_{\ell}\right)_{N}=\delta_{\ell}^{L} \delta_{m}^{M}\left(J_{\ell}\right)_{N}, \\
\sum_{\ell^{\prime} m^{\prime}}\left(V_{\ell m \ell^{\prime} m^{\prime}}^{L M}\right)_{N-1}^{\text {out }}\left(P_{\ell^{\prime} m^{\prime}}^{L M}\right)_{N}-\mathbb{R}_{\ell m}^{L M}\left(H_{\ell}\right)_{N-1}=\delta_{\ell}^{L} \delta_{m}^{M}\left(J_{\ell}\right)_{N-1}-\left(U_{\ell m}^{L M}\right)_{N-1}^{\text {out }} .
\end{array}\right.
$$

For each channel $L M$, the system of $2 C$ linear equations (B4) relates $C$ unknown solutions $\left(P_{\ell m}^{L M}\right)_{N}$ in the last grid point and $C$ values of the $\mathbb{R}_{\ell m}^{L M}$ matrix. Since two terms $\delta_{\ell}^{L} \delta_{m}^{M}\left(J_{\ell}\right)_{N}$ and $\delta_{\ell}^{L} \delta_{m}^{M}\left(J_{\ell}\right)_{N-1}$ in the right hand side of Eq. (B4) do never vanish simultaneously, this system is inhomogeneous at each step of iteration over the exchange potential. Solving Eq. (B4) for each channel $L M$ determines the complete $\mathbb{R}_{\ell m}^{L M}$ matrix and all values of the solutions in the last grid point $N$ in each channel $\left(P_{\ell m}^{L M}\right)_{N}$. Afterward, one can reconstruct the continuous solutions $\bar{P}^{L M}$ in the whole coordinate grid via outward vector sweep relation (A5) and renormalize them via transformation (9).

\section{APPENDIX C: NONITERATIVE PROCEDURE}

We redefine the partial harmonics of an electrostatic Coulomb potential (6) as follows:

$$
Y_{k}\left(P_{1}, P_{2}\right)=\operatorname{rr} y_{k}\left(P_{1}, P_{2}\right) .
$$

The harmonic (C1) of multiplicity $k$ satisfies the following differential equation: ${ }^{51}$

$$
\frac{\mathrm{d}^{2} Y_{k}\left(P_{1}, P_{2}\right)}{\mathrm{d} r^{2}}=\frac{k(k+1)}{r^{2}} Y_{k}\left(P_{1}, P_{2}\right)-\frac{(2 k+1)}{r} P_{1} P_{2},
$$

with the asymptotic conditions,

$$
\begin{aligned}
& Y_{k}\left(P_{1}, P_{2}\right)=A_{k} r^{k+1}(r \rightarrow 0) \quad \text { and } \\
& Y_{k}\left(P_{1}, P_{2}\right)=B_{k} r^{-k}(r \rightarrow \infty) .
\end{aligned}
$$

Even for the case of a simple diatomic molecule (such as $\mathrm{O}_{2}, \mathrm{~N}_{2}$, NO, and $\mathrm{CO}$ ), the exchange term (7) includes about $10^{5}$ potentials (6), which differs by (i) core shells (sum over index $c$ ), (ii) partial harmonics of core MOs (sums over indices $\ell_{c} m_{c}$ and $\ell_{c}^{\prime} m_{c}^{\prime}$ ), (iii) partial harmonics of a photoelectron (indices $\ell m$ and $\ell^{\prime} m^{\prime}$ ), and (iv) by multiplicity $k$. In order to reduce the amount of equations (C2), which must be solved, to a satisfactory order, we introduce the generalized harmonics for exchange Coulomb potential as described 
below. For this purpose we change the order of summations in the exchange term (7) entering Eq. (A1) as follows:

$$
\begin{aligned}
\sum_{\ell^{\prime} m^{\prime}} 2 K_{\ell m \ell^{\prime} m^{\prime}}^{e e} P_{\varepsilon \ell^{\prime} m^{\prime}} \\
=\sum_{c} 2 b_{c} \sum_{k q} \sum_{\ell_{c}^{\prime} m_{c}^{\prime}}(-1)^{m_{c}^{\prime}} \sqrt{\left(2 \ell_{c}^{\prime}+1\right)(2 \ell+1)} \\
\quad \times\left(\begin{array}{ccc}
\ell_{c}^{\prime} & k & \ell \\
0 & 0 & 0
\end{array}\right)\left(\begin{array}{ccc}
\ell_{c}^{\prime} & k & \ell \\
-m_{c}^{\prime} & q & m
\end{array}\right) \frac{Y_{k}\left(\bar{P}_{c}, \bar{P}_{\varepsilon}\right)}{r} P_{n_{c} \ell_{c}^{\prime} m_{c}^{\prime}} .
\end{aligned}
$$

Generalized potential $Y_{k}\left(\bar{P}_{c}, \bar{P}_{\varepsilon}\right)$ in Eq. (C4) describes the harmonic of multiplicity $k$ of the exchange Coulomb interaction of a photoelectron $\bar{P}_{\varepsilon}$ with the core electron $\bar{P}_{c}$ and is given via potentials $(\mathrm{C} 1)$ by

$$
\begin{aligned}
Y_{k}\left(\bar{P}_{c}, \bar{P}_{\varepsilon}\right)= & \sum_{\ell_{c} m_{c}} \sum_{\ell^{\prime} m^{\prime}}(-1)^{m_{c}} \sqrt{\left(2 \ell_{c}+1\right)\left(2 \ell^{\prime}+1\right)} \\
& \times\left(\begin{array}{ccc}
\ell_{c} & k & \ell^{\prime} \\
0 & 0 & 0
\end{array}\right)\left(\begin{array}{ccc}
\ell_{c} & k & \ell^{\prime} \\
-m_{c} & q & m^{\prime}
\end{array}\right) \\
& \times Y_{k}\left(P_{n_{c} \ell_{c} m_{c}}, P_{\varepsilon \ell^{\prime} m^{\prime}}\right) .
\end{aligned}
$$

Using Eq. (C2), it is straightforward to show that generalized potentials (C5) satisfy the following differential equation of the second order:

$$
\begin{aligned}
\frac{\mathrm{d}^{2} Y_{k}\left(\bar{P}_{c}, \bar{P}_{\varepsilon}\right)}{\mathrm{d} r^{2}}= & \frac{k(k+1)}{r^{2}} Y_{k}\left(\bar{P}_{c}, \bar{P}_{\varepsilon}\right) \\
& -\frac{(2 k+1)}{r} \sum_{\ell_{c} m_{c}} \sum_{\ell^{\prime} m^{\prime}}(-1)^{m_{c}} \\
& \times \sqrt{\left(2 \ell_{c}+1\right)\left(2 \ell^{\prime}+1\right)}\left(\begin{array}{ccc}
\ell_{c} & k & \ell^{\prime} \\
0 & 0 & 0
\end{array}\right) \\
& \left.\times\left(\begin{array}{ccc}
\ell_{c} & k & \ell^{\prime} \\
-m_{c} & q & m^{\prime}
\end{array}\right) P_{n_{c} \ell_{c} m_{c}} P_{\varepsilon \ell^{\prime} m^{\prime}}, \quad \text { C } 6\right)
\end{aligned}
$$

with the asymptotic conditions (C3). The complete exchange interaction of a photoelectron with a core of a simple diatomic molecule can now be described with the help of about $10^{2}$ generalized potentials (C5). In order to determine these potentials one needs to solve only about $10^{2}$ differential equations (C6) instead of about $10^{5}$ equations (C2) for potentials (6).

Let us now introduce the vector solution $\bar{P}$ that includes both the partial harmonic of a photoelectron and generalized potentials,

$$
\bar{P}=\left(\begin{array}{c}
\left\{P_{\ell m}\right\} \\
\left\{Y_{k}\left(\bar{P}_{c}, \bar{P}_{\varepsilon}\right)\right\}
\end{array}\right) .
$$

Unification of a system of differential equations for a photoelectron (Eq. (A1)) and generalized exchange potentials (Eq. (C6)) results in the following homogeneous system of differential equations relatively solutions (C7):

$$
\frac{\mathrm{d}^{2} \bar{P}}{\mathrm{~d} r^{2}}=\hat{F} \times \bar{P}
$$

with the $\hat{F}$ matrix defined by

$$
\hat{F}=\left(\begin{array}{ll}
\left\{F_{\ell m \ell^{\prime} m^{\prime}}\right\} & \left\{F_{\ell m c k}\right\} \\
\left\{F_{c k \ell^{\prime} m^{\prime}}\right\} & \left\{F_{c k c^{\prime} k^{\prime}}\right\}
\end{array}\right) .
$$

Matrix elements $F_{\ell m \ell^{\prime} m^{\prime}}$ are defined in Appendix A, and the explicit equations for the other matrix elements can be obtained with the help of Eqs. (C4) and (C6). They read as

$$
\begin{aligned}
F_{\ell m c k}= & \frac{2 b_{c}}{r} \sum_{q} \sum_{\ell_{c}^{\prime} m_{c}^{\prime}}(-1)^{m_{c}^{\prime}} \sqrt{\left(2 \ell_{c}^{\prime}+1\right)(2 \ell+1)} \\
& \times\left(\begin{array}{ccc}
\ell_{c}^{\prime} & k & \ell \\
0 & 0 & 0
\end{array}\right)\left(\begin{array}{ccc}
\ell_{c}^{\prime} & k & \ell \\
-m_{c}^{\prime} & q & m
\end{array}\right) P_{n_{c} \ell_{c}^{\prime} m_{c}^{\prime}},(\mathrm{C} 10)^{\prime} \\
F_{c k \ell^{\prime} m^{\prime}}= & -\frac{(2 k+1)}{r} \sum_{\ell_{c} m_{c}}(-1)^{m_{c}} \sqrt{\left(2 \ell_{c}+1\right)\left(2 \ell^{\prime}+1\right)} \\
& \times\left(\begin{array}{ccc}
\ell_{c} & k & \ell^{\prime} \\
0 & 0 & 0
\end{array}\right)\left(\begin{array}{ccc}
\ell_{c} & k & \ell^{\prime} \\
-m_{c} & q & m^{\prime}
\end{array}\right) P_{n_{c} \ell_{c} m_{c}}, \\
F_{c k c^{\prime} k^{\prime}=}= & \frac{k(k+1)}{r^{2}} \delta_{k k^{\prime}} \delta_{c c^{\prime} \cdot}
\end{aligned}
$$

Equations (C10) can be straightforwardly modified for the new solution function (C7) redefined via Eq. (12) after changing the integration variable according to Eq. (11), as shown in Sec. II B.

The homogeneous system of differential equations (C8) can be solved noniteratively by the numerical procedure described in Appendices A and B. In order to start numerical integration, the asymptotic conditions (C3) for generalized exchange potentials must be utilized. For a homogeneous system (i.e., $\bar{X}=0$ ), both $\bar{U}^{\text {out }}$ and $\bar{U}^{\text {in }}$ vectors in vector sweep relations (A5) must be omitted. In the discrete spectrum, matching the solutions integrated in the two opposite directions, and their derivations at the grid point $K$, result in a homogeneous system of linear equations (given by Eq. (B1) without right part) relatively to the values $\bar{P}_{K}$ of the desired vector function (C7) including both partial harmonics of a photoelectron and generalized exchange potentials. Equation (B2) is the criterion to determine the one-electron energy $\varepsilon$ in the discrete spectrum. In the continuum, integration must be performed outward only, and asymptotic conditions (8) and (C3) allow one to determine a complete $\mathbb{R}$ matrix, all values of the solution (C7) in the last grid point $\bar{P}_{N}$, as well as the asymptotic coefficients $B_{c k}$ in Eq. (C3), in each channel $L M$.

${ }^{1}$ J. K. Faegri and H. P. Kelly, Phys. Rev. A 19, 1649 (1979).

${ }^{2}$ J. A. Richards and F. P. Larkins, J. Phys. B 17, 1015 (1984).

${ }^{3}$ K. Zähringer, H.-D. Meyer, and L. S. Cederbaum, Phys. Rev. A 45, 318 (1992).

${ }^{4}$ K. Zähringer, H.-D. Meyer, and L. S. Cederbaum, Phys. Rev. A 46, 5643 (1992).

${ }^{5}$ B. Schimmelpfennig, B. M. Nestmann, and S. D. Peyerimhoff, J. Electron Spectrosc. Relat. Phenom. 74, 173 (1995). 
${ }^{6}$ I. Hjelte, L. Karlsson, S. Svensson, A. D. Fanis, V. Carravetta, N. Saito, M. Kitajima, H. Tanaka, H. Yoshida, A. Hiraya, I. Koyano, K. Ueda, and M. N. Piancastelli, J. Chem. Phys. 122, 084306 (2005).

${ }^{7}$ V. Carravetta and H. Ågren, Phys. Rev. A 35, 1022 (1987).

${ }^{8}$ H. Ågren and V. Carravetta, J. Chem. Phys. 87, 370 (1987).

${ }^{9}$ V. Carravetta, H. Ågren, O. Vahtras, and H. Jensen, J. Chem. Phys. 113, 7790 (2000)

${ }^{10}$ V. Averbukh and L. S. Cederbaum, J. Chem. Phys. 123, 204107 (2005).

${ }^{11}$ P. Kolorenč, V. Averbukh, K. Gokhberg, and L. S. Cederbaum, J. Chem. Phys. 129, 244102 (2008)

${ }^{12}$ V. Averbukh, Ph. V. Demekhin, P. Kolorenč, S. Scheit, S. Stoychev, A. I. Kuleff, Y. C. Chiang, K. Gokhberg, S. Kopelke, N. Sisourat, and L. S. Cederbaum, Interatomic electronic decay processes in singly and multiply ionized clusters, J. Electron Spectrosc. Relat. Phenom. (in press).

${ }^{13}$ R. E. Stratmann and R. R. Lucchese, J. Chem. Phys. 102, 8493 (1995).

${ }^{14}$ S. K. Botting and R. R. Lucchese, Phys. Rev. A 56, 3666 (1997).

${ }^{15}$ P. Lin and R. R. Lucchese, J. Chem. Phys. 116, 8863 (2002).

${ }^{16}$ S. Bonhoff, K. Bonhoff, B. Schimmelpfennig, and B. Nestmann, J. Phys. B 30, 2821 (1997).

${ }^{17}$ S. Bonhoff, K. Bonhoff, and K. Blum, J. Phys. B 32, 1139 (1999).

${ }^{18}$ H. Siegbahn, L. Asplund, and P. Kelfve, Chem. Phys. Lett. 35, 330 (1975).

${ }^{19}$ R. Fink, J. Electron Spectrosc. Relat. Phenom. 76, 295 (1995)

${ }^{20}$ R. Fink, J. Chem. Phys. 106, 4038 (1997).

${ }^{21}$ M. N. Piancastelli, M. Neeb, A. Kivimäki, B. Kempgens, H. M. Köppe, K. Maier, A. M. Bradshaw, and R. F. Fink, J. Phys. B 30, 5677 (1997).

${ }^{22}$ R. F. Fink, S. L. Sorensen, A. Naves de Brito, A. Ausmees, and S. Svensson, J. Chem. Phys. 112, 6666 (2000).

${ }^{23}$ R. F. Fink, M. N. Piancastelli, A. N. Grum-Grzhimailo, and K. Ueda, J. Chem. Phys. 130, 014306 (2009).

${ }^{24}$ S. K. Semenov, N. A. Cherepkov, G. H. Fecher, and G. Schönhense, Phys. Rev. A 61, 032704 (2000).

${ }^{25}$ S. K. Semenov and N. A. Cherepkov, J. Phys. B 36, 1409 (2003).

${ }^{26}$ S. K. Semenov, V. V. Kuznetsov, N. A. Cherepkov, P. Bolognesi, V. Feyer, A. Lahmam-Bennani, M. E. S. Casagrande, and L. Avaldi, Phys. Rev. A 75, 032707 (2007).

${ }^{27}$ D. M. Bishop, Adv. Quantum Chem. 3, 25 (1967).

${ }^{28}$ V. L. Sukhorukov, V. F. Demekhin, V. A. Yavna, I. D. Petrov, L. A. Demekhina, and S. V. Lavrentiev, Koord. Khim. (Sov.) 9, 158 (1983).

${ }^{29}$ V. L. Sukhorukov, S. V. Lavrentiev, V. F. Demekhin, and I. D. Petrov, Khim. Fiz. 3, 359 (1984)

${ }^{30}$ S. V. Lavrentiev, B. M. Lagutin, M. E. Vasil'eva, and V. L. Sukhorukov, Khim. Fiz. 7, 187 (1988).

${ }^{31}$ S. V. Lavrentiev, I. D. Petrov, B. M. Lagutin, and V. L. Sukhorukov, Khim. Fiz. 9, 81 (1990).

${ }^{32}$ S. V. Lavrentiev, M. E. Vasil'eva, I. D. Petrov, and V. L. Sukhorukov, Opt. Spektrosc. 69, 307 (1990).

${ }^{33}$ Y. F. Migal, Zhurnal Strukt. Khim. (Sov.) 17, 404 (1976).

${ }^{34}$ B. M. Lagutin and Y. F. Migal, Teor. i Eksper. Khim. (Sov.) 25, 12 (1989).

${ }^{35}$ V. A. Yavna, A. M. Nadolinskii, and V. F. Demekhin, Opt. Spektrosc. 69, 756 (1990).

${ }^{36}$ A. M. Nadolinskii, V. A. Yavna, A. N. Khoperskii, and D. V. Dzyuba, Opt. Spektrosc. 97, 854 (2004).

${ }^{37}$ A. Ehresmann, L. Werner, S. Klumpp, H. Schmoranzer, Ph. V. Demekhin, B. M. Lagutin, V. L. Sukhorukov, S. Mickat, S. Kammer, B. Zimmermann, and K. H. Schartner, J. Phys. B 37, 4405 (2004).

${ }^{38}$ A. Ehresmann, Ph. V. Demekhin, W. Kielich, I. Haar, M. A. Schlüter, V. L. Sukhorukov, and H. Schmoranzer, J. Phys. B 42, 165103 (2009).
${ }^{39} \mathrm{Ph}$. V. Demekhin, V. L. Sukhorukov, H. Schmoranzer, and A. Ehresmann, J. Chem. Phys. 132, 204303 (2010).

${ }^{40} \mathrm{Ph}$. V. Demekhin, D. V. Omel'yanenko, B. M. Lagutin, V. L. Sukhorukov, L. Werner, A. Ehresmann, K. H. Schartner, and H. Schmoranzer, Opt. Spektrosc. 102, 318 (2007).

${ }^{41}$ A. Ehresmann, L. Werner, S. Klumpp, S. Lucht, H. Schmoranzer, S. Mickat, R. Schill, K. H. Schartner, Ph. V. Demekhin, M. P. Lemeshko, and V. L. Sukhorukov, J. Phys. B 39, 283 (2006).

${ }^{42}$ A. Ehresmann, W. Kielich, L. Werner, Ph. V. Demekhin, D. V. Omel'yanenko, V. L. Sukhorukov, K. H. Schartner, and H. Schmoranzer, Eur. Phys. J. D 45, 235 (2007).

${ }^{43} \mathrm{Ph}$. V. Demekhin, I. D. Petrov, V. L. Sukhorukov, W. Kielich, P. Reiss, R. Hentges, I. Haar, H. Schmoranzer, and A. Ehresmann, Phys. Rev. A 80, 063425 (2009); Erratum: Phys. Rev. A 81, 069902(E) (2010).

${ }^{44} \mathrm{Ph}$. V. Demekhin, I. D. Petrov, T. Tanaka, M. Hoshino, H. Tanaka, K. Ueda, and W. Kielich, A. Ehresmann, J. Phys. B 43, 065102 (2010).

${ }^{45} \mathrm{Ph}$. V. Demekhin, I. D. Petrov, V. L. Sukhorukov, W. Kielich, A. Knie, H. Schmoranzer, and A. Ehresmann, Phys. Rev. Lett. 104, 243001 (2010).

${ }^{46} \mathrm{Ph}$. V. Demekhin, I. D. Petrov, V. L. Sukhorukov, W. Kielich, A. Knie, H. Schmoranzer, and A. Ehresmann, J. Phys. B 43, 165103 (2010).

${ }^{47}$ M. Y. Amusia and L. V. Chernysheva, ATOM-Computation of Atomic Structure and Processes (Nauka, Leninrad, 1983).

${ }^{48} \mathrm{P}$. G. Burke and M. J. Seaton, "Numerical solutions of the integrodifferential equations of electron-atom collision theory," in Atomic and Molecular Scattering, Methods in Computational Physics Vol. 10 (Academic, London, 1971), pp. 1-80.

${ }^{49}$ A. F. Starace, Theory of Atomic Photoionization, Handbuch der Physik (Springer, Berlin, 1982), Vol. 31.

${ }^{50}$ S. L. Sorensen, T. Åberg, J. Tulkki, E. Rachlew-Kallne, G. Sundstrom, and M. Kirm, Phys. Rev. A 50, 1218 (1994).

${ }^{51}$ K. Smith, R. J. W. Henry, and P. G. Burke, Phys. Rev. 147, 21 (1966).

${ }^{52}$ H. Liebel, R. Müller-Albrecht, S. Lauer, F. Vollweiler, A. Ehresmann, and H. Schmoranzer, J. Phys. B 34, 2581 (2001).

${ }^{53}$ R. C. Wu, J. Quant. Spectrosc. Radiat. Transf. 37, 1 (1987).

${ }^{54}$ D. Čubrič, A. A. Wills, J. Comer, and M. Ukai, J. Phys. B 26, 3081 (1993).

${ }^{55}$ H. Wang, R. F. Fink, M. N. Piancastelli, I. Hjelte, K. Wiesner, M. Bässler, R. Feifel, O. Björneholm, C. Miron, A. Giertz, F. Burmeister, S. L. Sorensen, and S. Svensson, J. Phys. B 34, 4417 (2001).

${ }^{56}$ K. C. Prince, M. Vondráček, J. Karvonen, M. Coreno, R. Camilloni, L. Avaldi, and M. de Simone, J. Electron Spectrosc. Relat. Phenom. 101-103, 141 (1999).

${ }^{57}$ B. Kempgens, A. Kivimäki, M. Neeb, H. M. Koppe, A. M. Bradshaw, and J. Feldhaus, J. Phys. B 29, 5389 (1996).

${ }^{58}$ H. Liebel, A. Ehresmann, H. Schmoranzer, Ph. V. Demekhin, B. M. Lagutin, and V. L. Sukhorukov, J. Phys. B 35, 895 (2002).

${ }^{59}$ D. M. P. Holland, D. A. Shaw, S. M. McSweeney, M. A. MacDonald, A. Hopkirk, and M. A. Hayes, Chem. Phys. 173, 315 (1993).

${ }^{60}$ E. Kukk, J. D. Bozek, W. Cheng, R. F. Fink, A. A. Wills, and N. Berrah, J. Chem. Phys. 111, 9642 (1999).

${ }^{61}$ F. K. Gel'mukhanov, L. N. Mazalov, and A. V. Kondratenko, Chem. Phys. Lett. 46, 133 (1977).

${ }^{62}$ Z. W. Gortel, R. Teshima, and D. Menzel, Phys. Rev. A 58, 1225 (1998).

${ }^{63}$ H. Wang, R. F. Fink, M. N. Piancastelli, M. Bässler, I. Hjelte, O. Björneholm, F. Burmeister, R. Feifel, A. Giertz, C. Miron, S. L. Sorensen, K. Wiesner, and S. Svensson, Chem. Phys. 289, 31 (2003).

${ }^{64}$ A. Cesar and H. Ågren, Phys. Rev. A 45, 2833 (1992). 